\title{
Cyclosporine A attenuates cardiac dysfunction induced by sepsis via inhibiting calcineurin and activating AMPK signaling
}

\author{
JINGMIAO LIU, DA CHEN, XIAOWEI LIU and ZHI LIU \\ Department of Emergency Medicine, The First Affiliated Hospital of China \\ Medical University, Shenyang, Liaoning 110001, P.R. China
}

Received December 24, 2015; Accepted January 26, 2017

DOI: $10.3892 / \mathrm{mmr} .2017 .6421$

\begin{abstract}
The aim of the present study was to investigate whether cyclosporine A (CSA) improved cardiac dysfunction at an early stage of sepsis. Male Wistar rats were randomly divided into the following three groups: the sham-operated control group, the cecal ligation puncture (CLP) procedure-induced sepsis group and the CSA intervention group. Cecal ligation was performed to generate a sepsis model. At different time points $(2,6,12,24$ and $72 \mathrm{~h}$ ) following sepsis induction, blood pressure, cardiac function, and non-esterified free fatty acid (NEFA) levels in the plasma and myocardia were measured, and the expression levels of components associated with the AMP-activated protein kinase (AMPK)-acetyl CoA carboxylase (ACC)-carnitine palmitoyl transferase 1 (CPT1) signaling pathway were compared among the three groups. Sepsis induced a decrease in blood pressure and cardiac function at $24 \mathrm{~h}$ following sepsis induction in the CLP group, and CSA treatment ameliorated these pathophysiological alterations. In addition, rats in the CLP group exhibited significant increases in calcineurin activity and NEFA accumulation in the heart when compared with those in the sham group. These effects were attenuated by CSA treatment. Mechanistically, the activity of the AMPK-ACC-CPT1 pathway was enhanced by CSA treatment. The present study revealed that CSA treatment increases cardiac function at an early stage of sepsis in rats. This treatment partially suppresses calcineurin activity while activating the AMPK-TCC-CPT1 pathway.
\end{abstract}

Correspondence to: Dr Zhi Liu, Department of Emergency Medicine, The First Affiliated Hospital of China Medical University, 155 North of Nanjing Street, Heping, Shenyang, Liaoning 110001, P.R. China

E-mail: liuzhicmu@163.com

Key words: Sepsis, cyclosporine A, calcineurin, free fatty acid, AMP-activated protein kinase-acetyl CoA carboxylase-carnitine palmitoyl transferase 1 pathway

\section{Introduction}

Sepsis is associated with a high level of mortality worldwide and is frequently associated with the functional failure of one or more organs $(1,2)$. In the cardiovascular system, it is well known that sepsis induces myocardial dysfunction and heart failure, which is characterized by a significant decrease in cardiac output (1). In a clinical setting, cardiac dysfunction induced by sepsis accounts for $\sim 15 \%$ of all cases of mortality, thus it is closely associated with the prognosis of sepsis $(3,4)$. Therefore, it is critical to improve cardiac function in order to increase survival rates during sepsis, particularly at the early stages. Clinical studies have indicated that sepsis leads to functional depression of the heart without severe structural damage $(2,5)$, which suggests that sepsis-associated myocardial dysfunction may be reversible. Despite previous studies investigating sepsis-induced pathological alterations in human and animal models $(1,6)$. the precise mechanisms by which sepsis induces myocardial depression during the early stages remains unknown. However, a number of molecules and signaling pathways, including those involved in oxidative stress, mitochondrial damage and energy metabolism disorders, have been proposed to be involved (4).

Calcineurin, a $\mathrm{Ca}^{2+}$-calmodulin-activated serine-threonine phosphatase, serves an important role in cardiovascular physiology and pathophysiology (7). Overexpression of calcineurin in cardiomyocytes regulated by the myosin heavy chain promoter has been demonstrated to promote cardiac hypertrophy (8), which is reversible (9). In addition, inhibition of calcineurin by specific inhibitors, such as cyclosporine and FK506, protected the heart from pressure overload-induced cardiac hypertrophy (10). A previous study indicated that the activity of calcineurin was increased in cardiomyocytes upon exposure to lipopolysaccharides, one of the predominant molecule types released following sepsis (11). These molecules promote pathological alterations in sepsis-associated myocardial dysfunction (12), indicating a potential role for calcineurin in the myocardial depression induced by sepsis. Cyclosporine A (CSA), an immunosuppressant and calcineurin inhibitor, has been widely used in the clinic to suppress immune responses against transplanted organs. However, to date, a relatively small number of studies have been performed to examine the efficacy of CSA in the treatment of sepsis, and the results generated have been controversial. One study in 
particular demonstrated that CSA served a protective role in rats suffering from sepsis-induced acute kidney injury (13), however, it was later reported to exacerbate mortality in a murine model of septic shock (14). Previous studies have revealed that calcineurin is involved in sepsis-associated myocardial dysfunction (15), and that this dysfunction is improved by CSA treatment in animal models (16). However, the mechanisms underlying efficient CSA treatment are not fully understood.

During sepsis, free fatty acid metabolic disorders, such as those involving non-esterified free fatty acids (NEFA), and the misregulation of hormones and cytokines have been observed to occur (17). The AMP-activated protein kinase (AMPK)-acetyl CoA carboxylase (ACC)-carnitine palmitoyl transferase 1 (CPT1) signaling pathway is one of the pathways involved in mediating myocardial energy metabolism $(18,19)$. The AMPK-ACC-CPT1 pathway is activated in response to hormone signaling and stressors, including low blood glucose levels, hypoxia and ischemia (20). A previous study demonstrated that elevated calcineurin activity interferes with AMPK-ACC-CPT1 pathway function, whereas CSA ameliorated fatty acid metabolism by inhibiting calcineurin activity in rodent hippocampi (3). However, whether the AMPK-ACC-CPT1 signaling pathway contributes to the sepsis-induced alterations in the lipid metabolism of the heart remains unclear.

The aim of the present study was to determine whether CSA might be an effective treatment for myocardial repression during the early stages of the septic process using a rodent model of sepsis. In addition, the present study investigated whether the AMPK-ACC-CPT1 pathway may be involved in CSA-mediated protection against sepsis-associated functional damage of the myocardium.

\section{Materials and methods}

Animals. A total of 135 male Wistar rats (age, 4-6 weeks; weight, 250-300 g). were purchased from the Experimental Animal Center of China Medical University (Shenyang, China). The rats, 3 per cage, were maintained at temperature at $22^{\circ} \mathrm{C}, 45 \%$ humidity, and $12 \mathrm{~h}$ light/dark cycle, having free access to food and water.

All animal experiments were approved by the Institutional Animal Care and Use Committee of the Medical Ethics Committee of The First Hospital of China Medical University (Beijing, China).

Materials. CSA (Pharmaceuticals AG, Rothreuz ZG, Switzerland), a calcineurin activity measurement kit (Nanjing Jiancheng Bioengineering Institute, Nanjing, China), a free fatty acid assay kit (Nanjing Jiancheng Bioengineering Institute), a bicinchoninic acid (BCA) assay kit (Beyotime Institute of Biotechnology, Haimen, China), and primary antibodies against AMPK (cat. no. sc-25792), phosphorylated (p)-AMPK (cat. no. sc-33524), ACC- $\beta$ (cat. no. sc-26822), p-ACC- $\beta$ (cat. no. sc-30446-R), CPT1 (cat. no. sc-139482) and $\beta$-actin (cat. no. sc-47778, all from Santa Cruz Biotechnology Inc., Dallas, TX, USA) were used in the present study. In addition, $10 \%$ chloral hydrate, phosphate-buffered saline, TBS solution and Tween-20 were procured from Sinopharm Chemical Reagent Co., Ltd. (Shanghai, China).

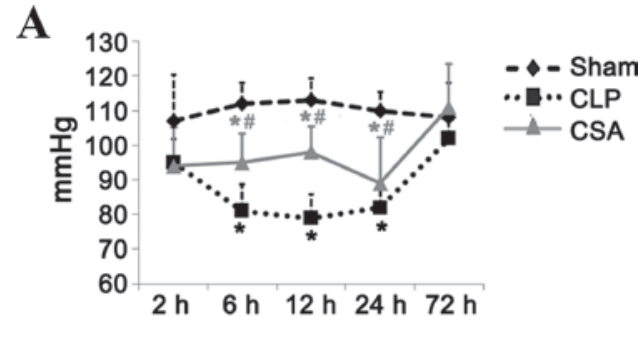

B

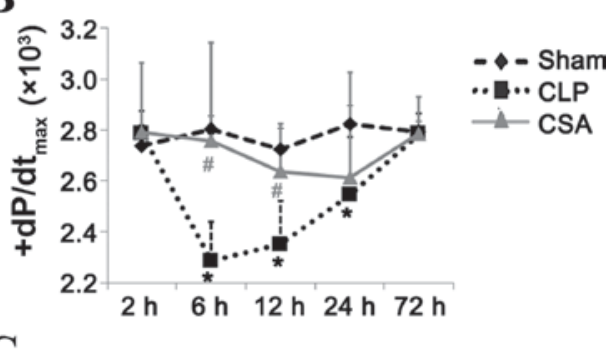

C

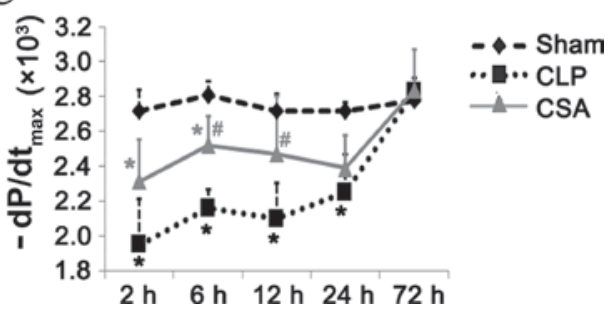

Figure 1. CSA significantly attenuated the sepsis-induced decrease in blood pressure and cardiac function. (A) CSA inhibited the sepsis-induced decrease in blood pressure in male Wistar rats. Blood pressure was measured at 2, 6, 12 , 24 and $72 \mathrm{~h}$ following establishment of the sepsis model. CSA improved the (B) $+\mathrm{dP} / \mathrm{dt}_{\max }$ and $(\mathrm{C})-\mathrm{dP}^{\mathrm{d} \mathrm{dt}_{\max }}$ values in rats with sepsis at 6 and $12 \mathrm{~h}$ when compared with the CLP group thereby ameliorating sepsis-induced cardiac dysfunction. Data are presented as the mean \pm standard deviation $(n=6)$. ${ }^{*} \mathrm{P}<0.05$ vs. sham; ${ }^{\text {P }}<0.05$ vs. CLP. Sham, sham-operated rats; CLP, cecal ligation puncture procedure sepsis model group; CSA, cyclosporine A-treated sepsis model group; $+\mathrm{dP} / \mathrm{dt}_{\max }$, maximal rate of left ventricle systolic pressure rise; $-\mathrm{dP} / \mathrm{dt}_{\max }$, the maximal rate of the left ventricle diastolic pressure decrease.

Generation of the septic animal model using the cecal ligation puncture $(C L P)$ procedure. Rats were anesthetized with a subcutaneous injection of $10 \%$ chloral hydrate $(4 \mathrm{ml} / \mathrm{kg})$, fixed on a $22^{\circ} \mathrm{C}$ warm pad and a section of abdominal skin was sterilized. A ventral midline incision of $\sim 1.5 \mathrm{~cm}$ was made, followed by cecal ligation at one third of the distance from the end of the cecum. The cecum was pierced twice with a needle (no. 18) and fecal overflow was promoted by squeezing. The cecum was then ligated and placed back in the abdominal cavity before the opening was closed. A total of $5 \mathrm{ml}$ saline was immediately injected into the abdomen to prevent shock.

Animal grouping. The rats were randomly divided into the following 3 groups: the control group (sham) where an abdominal incision was performed without cecal ligation; the sepsis group (CLP group), whereby abdominal incision and cecal ligation were performed; the CSA intervention group (CSA group), whereby $10 \mathrm{mg} / \mathrm{kg}$ CSA was administered by intraperitoneal injection at $30 \mathrm{~min}$ following anesthesia, which was followed by abdominal incision and cecal ligation. Each group was observed at 2, 6, 12, 24 and $72 \mathrm{~h}$ following the operation. Rats that did not survive over course of the study 
were excluded (control, 0; CLP, 23; CSA, 19) from the sample collection and data analysis.

Determination of cardiac function. Cardiac function was measured via catheterization. Briefly, each rat was anesthetized with $10 \%$ chloral hydrate $(4 \mathrm{ml} / \mathrm{kg}$, i.p.), fixed with the strip wrapped around the limbs on a supine position on a small operation table, and the right common carotid artery was exposed. The proximal end of the catheter, which was connected to a computer monitored by a Biopac polygraph (Biopac UK Ltd., Pershore, UK), was inserted into the left ventricle via the proximal end of the right common carotid artery. The full procedure was guided by the computer-aided imaging system AcqKnowledge 4.0 (Biopac UK Ltd.). Following several min of stabilization, the left ventricular end-diastolic pressure, the maximal rate of left ventricle systolic pressure rise $\left(+\mathrm{dP} / \mathrm{dt}_{\max }\right)$ and the maximal rate of the left ventricle diastolic pressure decrease $\left(-\mathrm{dP} / \mathrm{dt}_{\max }\right)$ were measured using the Biopac polygraph. Rat cardiac function was measured at 2, 6, 12, 24 and $72 \mathrm{~h}$ following the operation.

Blood sample collection. After the rats were anesthetized, blood samples were collected from the carotid artery using an arterial clamp at 2, 6, 12, 24 and $72 \mathrm{~h}$ following the operation. The collected blood $(5 \mathrm{ml})$ was centrifuged at $1,566 \times \mathrm{g}$ for $10 \mathrm{~min}$ at room temperature and the supernatant was immediately frozen at $-80^{\circ} \mathrm{C}$ for future use.

Determination of the plasma and cardiac tissue homogenate index. Following blood sample collection, the rat chest was immediately opened, the heart was isolated and the left ventricle was removed and immediately frozen at $-80^{\circ} \mathrm{C}$. The cardiac calcineurin activity assay, NEFA concentration determination, and the BCA protein quantification assay were performed based on the protocols provided by the manufacturers. Briefly, left ventricles from rats were collected and snap-frozen at $-80^{\circ} \mathrm{C}$. For protein purification, the heart tissues were homogenized in RIPA buffer $(25 \mathrm{mM}$ Tris $\bullet \mathrm{HCl}$ $\mathrm{pH} 7.6,150 \mathrm{mM} \mathrm{NaCl}, 1 \% \mathrm{NP}-40,1 \%$ sodium deoxycholate, $0.1 \%$ SDS) and protease inhibitors PMSF (ST505, Beyotime Institute of Biotechnology, Haimen, China were added prior to the purification. Protein concentrations were determined with a BCA kit (Beyotime Institute of Biotechnology). cardiac calcineurin activity assays were performed with a calcineurin activity assay kit (according to the protocol provided by the manufacturers. NEFA concentrations were determined with a commercial kit.

Oil red $O$ staining. Oil red $\mathrm{O}$ staining was performed as previously described $(21,22)$ with some modifications. Briefly, frozen myocardial tissues were sectioned to produce 8 - $\mu$ m-thick tissue slices. The sections were fixed in $4 \%$ formaldehyde for $10 \mathrm{~min}$ at room temperature. The slides were then rinsed with distilled water and dried, before they were by incubated with Oil red $\mathrm{O}$ dye (Oil red, $0.5 \mathrm{~g}$; dissolved in $60 \%$ isopropronol) at $37^{\circ} \mathrm{C}$ for $20 \mathrm{~min}$. The slides were subsequently submersed in $60 \%$ isopropanol for $10 \mathrm{~s}$, washed with water, and stained with $0.5 \%$ hematoxylin for $30 \mathrm{sec}$ at room temperature. The slides were mounted in glycerogelatin and sealed.
Western blot analysis. Western blotting was performed to determine the expression levels of AMPK, p-AMPK, ACC, p-ACC and CPT1 in the myocardium. Briefly, proteins were extracted from the myocardial tissues using a protein extraction reagent (Beyotime Institute of Biotechnology) and protein concentration was measured using the BCA Assay kit (Beyotime Institute of Biotechnology). Heat-denatured protein $(60 \mu \mathrm{g})$ was loaded onto a 10\% SDS-PAGE gel, followed by protein transfer to a nitrocellulose membrane. The membrane was subsequently blocked with 5\% non-fat dried milk for $1 \mathrm{~h}$ at room temperature, before it was probed overnight with the diluted primary antibody of interest (AMPK, 1:500; p-AMPK, 1:500; ACC, 1:1,000; p-ACC, 1:1,000; CPT1, 1:500) at $4^{\circ} \mathrm{C}$. The PVDF membrane was then washed with TBS-Tween solution and incubated with horseradish peroxidase-conjugated goat anti-rabbit or rabbit anti-mouse immunoglobulin G (1:10,000; OriGene Technologies, Beijing, China) at room temperature for $1 \mathrm{~h}$. Actin expression levels were used as a control. Protein bands were visualized using an enhanced chemiluminescence developer (MicroChemi, DNR, Israel) and relative densitometry was performed using ImageJ version 1.63 (imagej.nih. gov/ij/).

Statistical analysis. Data are expressed as the mean \pm standard deviation. Statistical significance was analyzed using SPSS statistical software (version, 19.0; IBM SPSS, Armonk, $\mathrm{NY}$, USA). Independent sample $t$-tests were used for comparing the statistical significance between two groups. $\mathrm{P}<0.05$ was considered to indicate a statistically significant difference.

\section{Results}

CSA significantly attenuated the sepsis-induced decrease in blood pressure and cardiac function. As expected, sepsis induced a significant decrease in blood pressure at 6, 12 and $24 \mathrm{~h}$ in the CSA group, respectively, when compared with the CLP group $(\mathrm{P}<0.05$; Fig. 1A). At $72 \mathrm{~h}$ following the induction of sepsis, the blood pressure of the CLP rats recovered to the same level as that observed in the sham group, suggesting that this decrease in blood pressure occurred at an early stage in the septic model. CSA treatment significantly attenuated this decrease at 6 and $12 \mathrm{~h}$; however, not at $24 \mathrm{~h}$ when compared with the CLP group ( $\mathrm{P}<0.05$; Fig. 1A). Sepsis-induced cardiac dysfunction was evident at $6 \mathrm{~h}$ and persisted for up to $24 \mathrm{~h}$, as demonstrated by the decreased $+\mathrm{dP} / \mathrm{dt}_{\max }$ and $-\mathrm{dP} / \mathrm{dt}_{\max }$ values in the CLP group when compared with the sham group (Fig. 1B and C). The CSA group demonstrated significantly improved cardiac function when compared with the CLP group at 6 and $12 \mathrm{~h}$ following the operation $\left(+\mathrm{dP} / \mathrm{dt}_{\max }, \mathrm{P}<0.05\right.$ at both time points; $-\mathrm{dP} / \mathrm{dt}_{\max }, \mathrm{P}<0.05$ at both time points; Fig. $1 \mathrm{~B}$ and $\mathrm{C}$ ). At $72 \mathrm{~h}$ following the induction of sepsis, no significant difference was observed between the $+\mathrm{dP} / \mathrm{dt}_{\max }$ and $-\mathrm{dP} / \mathrm{dt}_{\max }$ values of the CLP and CSA groups when compared with those of the sham group (Fig. 1B and C). Therefore, this sepsis model may facilitate the study of the effects of sepsis on myocardial function during the early stages of sepsis. The results demonstrated that CSA administration efficiently attenuated the decrease in blood pressure and cardiac function typically induced by sepsis. 
Table I. CSA significantly inhibited the sepsis-induced increase in calcineurin activity in the myocardium.

\begin{tabular}{lccccc}
\hline Group & $2 \mathrm{~h}$ & $6 \mathrm{~h}$ & $12 \mathrm{~h}$ & $24 \mathrm{~h}$ & $72 \mathrm{~h}$ \\
\hline Sham (unit/mg protein) & $0.67 \pm 0.042$ & $0.57 \pm 0.037$ & $0.37 \pm 0.058$ & $0.5 \pm 0.046$ & $0.52 \pm 0.074$ \\
CLP (unit/mg protein) & $0.77 \pm 0.096$ & $0.93 \pm 0.076^{\mathrm{a}}$ & $1.38 \pm 0.077^{\mathrm{a}}$ & $1.6 \pm 0.085^{\mathrm{a}}$ & $1.12 \pm 0.102^{\mathrm{a}}$ \\
CSA (unit/mg protein) & $0.61 \pm 0.069$ & $0.59 \pm 0.056^{\mathrm{b}}$ & $0.87 \pm 0.059^{\mathrm{b}}$ & $1.04 \pm 0.072^{\mathrm{b}}$ & $1.03 \pm 0.06$ \\
\hline
\end{tabular}

Calcineurin activity was measured at different time points following induction of sepsis. Data are expressed as the mean \pm standard deviation ( $\mathrm{n}=6)$. ${ }^{\mathrm{P}} \mathrm{P}<0.05$ vs. sham; ${ }^{\mathrm{P}} \mathrm{P}<0.05$ vs. CLP. Sham, sham-operated rats; CLP, cecal ligation puncture procedure sepsis model group; CSA, cyclosporine A-treated sepsis model group.

A

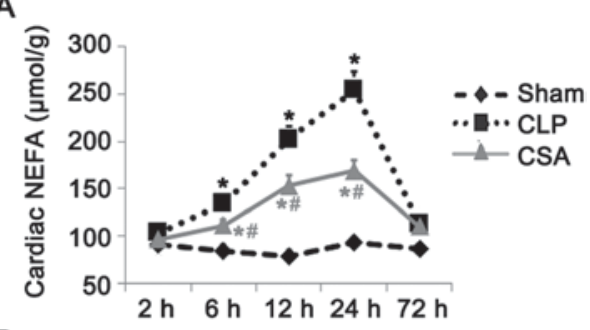

B

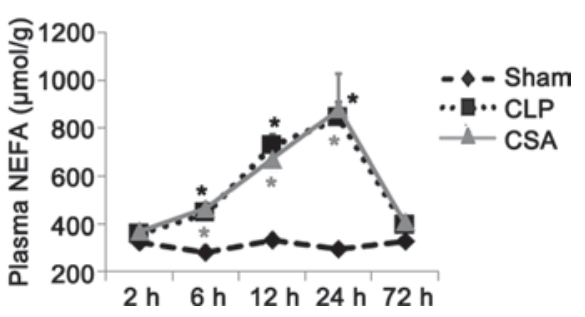

Figure 2. CSA significantly decreased the sepsis-induced increase NEFA levels in the myocardium but not in the serum. (A) CSA significantly reduced the NEFA concentration in the myocardium between 2 and $24 \mathrm{~h}$ when compared with the CLP group, but failed to restore them to normal levels. (B) CSA did not significantly affect the NEFA levels in serum when compared with the CLP group. Data are presented as mean \pm standard deviation $(n=6)$. ${ }^{*} \mathrm{P}<0.05$ vs. sham; ${ }^{\prime} \mathrm{P}<0.05$ vs. CLP. NEFA, non-esterified fatty acids; sham, sham-operated rats; CLP, cecal ligation puncture procedure sepsis model group; CSA, cyclosporine A-treated sepsis model group.

CSA significantly inhibited the increased activity of calcineurin by sepsis in the myocardium. As CSA is a specific inhibitor of calcineurin, and calcineurin has been implicated in the maintenance of cardiac homeostasis (23), the present study investigated whether sepsis may alter the activity of calcineurin in the myocardium. As shown in Table I, calcineurin activity was significantly higher in the CLP group when compared with the sham group at $6,12,24$ and $72 \mathrm{~h}$ following induction of sepsis ( $\mathrm{P}<0.05$, CLP group vs. the sham group). However, CSA treatment significantly inhibited the increase in calcineurin activity at 6,12 and $24 \mathrm{~h}$ when compared with the CSP group $(\mathrm{P}<0.05$; Table I). No significant difference in calcineurin levels were observed in the CSA group when compared with the CLP group at $72 \mathrm{~h}$ (Table I). These results indicated that sepsis is associated with elevated calcineurin activity in the myocardium, which may be suppressed by CSA treatment.

CSA significantly decreased the sepsis-induced increase in NEFA levels in the myocardium but not in the serum. It has been previously reported that sepsis alters energy metabolism in tissues and organs (5). Therefore, the authors of the present study investigated whether cardiac lipid metabolism was altered in the rat model of sepsis. As the levels of NEFA are a useful indicator of altered lipid oxidation, the concentration of NEFA in the myocardium and plasma of rats with and without sepsis was measured. As shown in Fig. 2, the levels of NEFA were significantly elevated in the plasma and myocardium at 6, 12 and $24 \mathrm{~h}$ in the CLP group when compared with those in the sham group $(\mathrm{P}<0.05)$. Although CSA treatment was associated with a significant increase in NEFA concentration in the myocardium at 6,12 and $24 \mathrm{~h}$ when compared with the sham group, no significant difference in plasma NEFA concentrations was observed in the CSA group when compared with the CLP group at any time point (Fig. 2). Thus, CSA treatment substantially diminished the levels of NEFA in the myocardium but not in the serum of rats with sepsis.

To confirm the altered levels of NEFA in the myocardium of rats in the three experimental groups, the myocardia were stained using Oil red O. The sham group exhibited no obvious Oil red $\mathrm{O}$ staining at any of the time points examined (Fig. 3A and $\mathrm{B}$, and data not shown). As early as $2 \mathrm{~h}$ following sepsis induction, the myocardia in the CLP group exhibited marked red staining, which peaked at $24 \mathrm{~h}$ and persisted for at least $72 \mathrm{~h}$ (Fig. 3C-G). Despite positive red staining in the myocardia of the CSA group, the intensity of red staining was lower when compared with that of the CLA group at each time point examined (Fig. 3H-L). Oil red $\mathrm{O}$ staining reflects free fatty acid accumulation in the myocardium. Therefore, these results indicate that CSA may facilitate free fatty acid metabolism in the myocardium, thus reducing the accumulation induced by sepsis.

CSA treatment activated the AMPK-ACC-CPT1 signaling pathway. In order to investigate whether sepsis induced alterations in lipid metabolism, the present study investigated whether the AMPK-ACC-CPT1 signaling pathway may be involved. The AMPK-ACC-CPT1 pathway has been proposed to be an important regulator of lipid metabolism in the heart (24). Therefore, the expression levels of AMPK, ACC and CPT1 in the heart tissues from rats in all three experimental groups were analyzed by western blotting. As shown in Fig. 4, the protein expression levels of AMPK and ACC did not exhibit any significant differences among the three groups at 6,24 and $72 \mathrm{~h}$ time points. However, the active forms of the two proteins, p-AMPK and p-ACC, were significantly elevated in the CLP group when compared with the sham group at all time points 

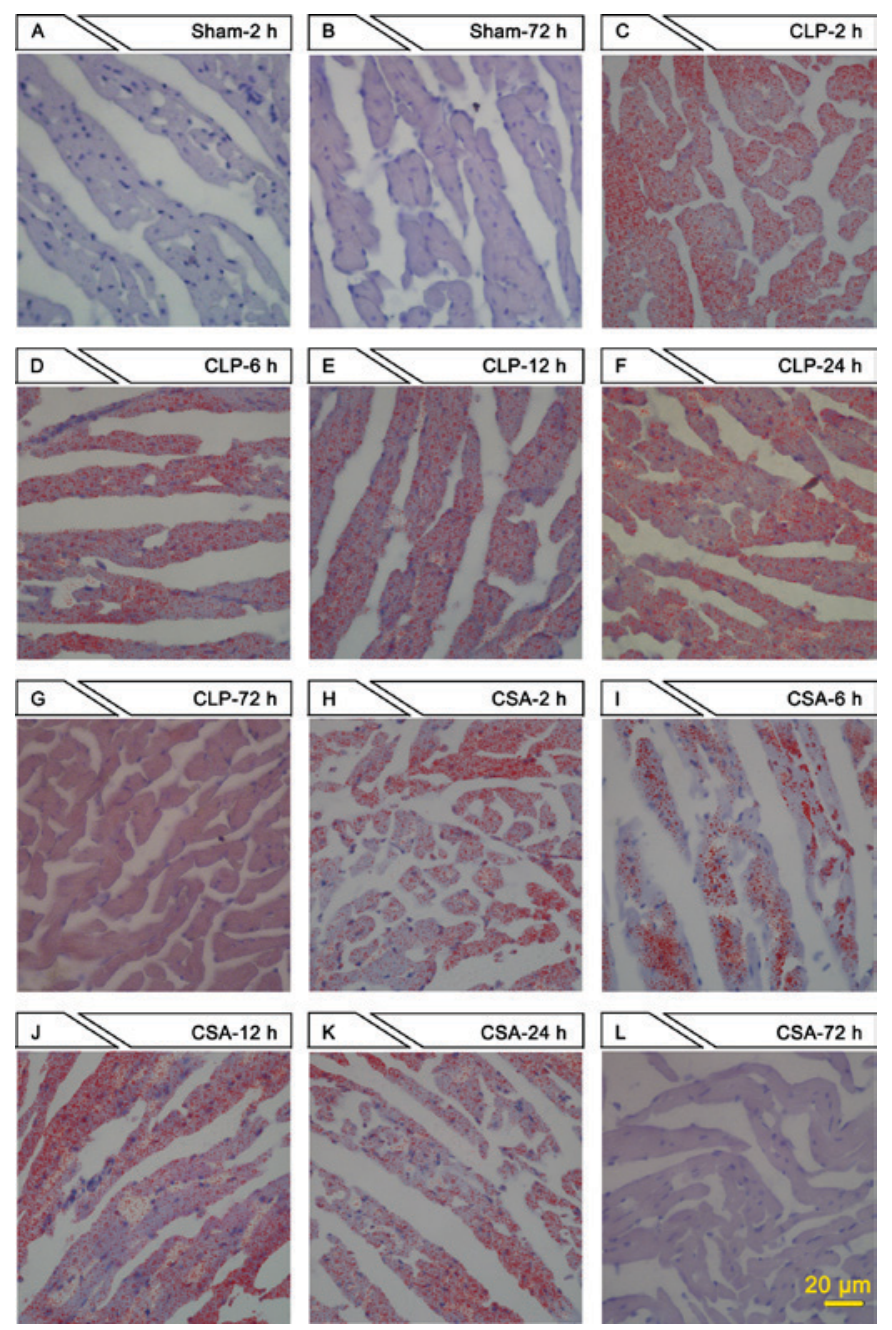

Figure 3. CSA significantly decreased NEFA accumulation in the myocardium. Oil red $\mathrm{O}$ staining was performed on heart tissue sections prepared from rats in sham, CLP and CSA groups at different time points $(2,6,12,24$ and $72 \mathrm{~h}$ ). Representative images are shown (scale bar, $20 \mu \mathrm{m}$; magnification, $\mathrm{x} 400$ ). Sham group at (A) $2 \mathrm{~h}$ and (B) $72 \mathrm{~h}$; CLP group at (C) $2 \mathrm{~h}$, (D) $6 \mathrm{~h}$, (E) $12 \mathrm{~h},(\mathrm{~F}) 24 \mathrm{~h}$ and $(\mathrm{G}) 72 \mathrm{~h}$; CSA group at (H) $2 \mathrm{~h}$, (I) $6 \mathrm{~h},(\mathrm{~J}) 12 \mathrm{~h}$, (K) $24 \mathrm{~h}$ and (L) $72 \mathrm{~h}$. NEFA, non-esterified fatty acids; sham, sham-operated rats; CLP, cecal ligation puncture procedure sepsis model group; CSA, cyclosporine A-treated sepsis model group.

( $\mathrm{P}<0.05$; Fig. 4). In addition, CPT1 expression was elevated in the CLP group when compared with the sham group at all time points $(\mathrm{P}<0.05$; Fig. 4). CSA treatment increased the activity of p-AMPK, p-ACC and CPT1 at 6 , and $24 \mathrm{~h}$, when compared with the CLP and sham groups (Fig. 4, P<0.05). Collectively, these data suggest that sepsis may increase the activity of the AMPK-ACC-CPT1 signaling pathway, and CSA may further enhance the activation of this pathway by increasing the levels of the active, phosphorylated forms or the total protein levels of these components.

\section{Discussion}

Hemodynamic alterations serve a crucial role in the occurrence and development of shock and multiple organ dysfunction syndrome in the pathophysiology of sepsis (25). In particular, left ventricular function is an important contributor to hemodynamic alterations, and significantly affects the prognosis of sepsis (26). A previous study demonstrated that early sepsis may lead to decreased heart function, which is attributable to myocardial ischemia and hypoxia as well as altered myocardial energy metabolism (27).

The present study investigated how early stage sepsis affected cardiac function and whether CSA may be able to exert any significant protective effects. The sepsis model induced a decrease in blood pressure and impaired cardiac function; the latter of which was demonstrated by decreased $+\mathrm{dP} / \mathrm{dt}_{\max }$ and $-\mathrm{dP} / \mathrm{dt}_{\max }$ values at 6 and $24 \mathrm{~h}$ following sepsis induction. However, these functional indices returned to levels comparable to those of the sham group, suggesting that the sepsis model applied in the current study may be a short-term model that is only appropriate for studying the effects of early-stage sepsis on the heart. In addition, CSA treatment was observed to substantially improve blood pressure and cardiac function at 6 and $12 \mathrm{~h}$ following sepsis induction. Thus, CSA may be an effective drug in facilitating the treatment of sepsis at the early stages of infection, in order to ameliorate cardiac function.

Previously, CSA has been widely used as an immunosuppressant to repress immune responses against transplanted organs in the clinic (28). However, previous studies have indicated that CSA is a specific inhibitor of calcineurin; a calcium-sensitive phosphatase, which serves a well-defined role in cardiac physiology and pathophysiology $(12,15)$. In addition, calcineurin may be involved in sepsis-induced cardiac dysfunction $(12,15)$. The present study demonstrated that calcineurin activity was elevated following induction of sepsis. This elevated activity of calcineurin was repressed by CSA treatment. In the CLP group, calcineurin activity was significantly increased between 6 and $72 \mathrm{~h}$ following sepsis induction, and CSA treatment efficiently suppressed this increase during the $6-24 \mathrm{~h}$ period. This coincided with cardiac functional recovery at 6 to $24 \mathrm{~h}$ by CSA treatment. The results indicated that CSA treatment may alleviate the cardiac dysfunction induced by sepsis, in part, through suppression of calcineurin activity. Cardiac function was normal in the CLP group at $72 \mathrm{~h}$. However, at this time point, calcineurin activity remained significantly higher in the CLP group when compared with the sham group. Therefore, it is possible that additional mechanisms, other than calcineurin activity, may be involved in mediating myocardial repression during the early stages of sepsis. Alternatively, it is possible that the elevated calcineurin levels at $72 \mathrm{~h}$ may not have been sufficient to induce cardiac dysfunction.

Sepsis-induced alterations in myocardial energy metabolism serve an important role in the development of cardiac dysfunction (29). Altered myocardial energy metabolism is characterized by impaired mitochondrial function, reduced lipid metabolism and glucose metabolism disorders in patients and mouse models (20). Myocardial NEFA accumulation is an important manifestation of myocardial metabolic dysregulation (20). Under physiological conditions, cardiomyocytes do not synthesize and store a significant quantity of NEFA; instead, myocardial cells take up NEFA from the circulating serum. Following uptake, NEFA is predominantly transported to the mitochondria to provide energy, and to participate in triglyceride and lysophospholipid synthesis. Myocardial energy is primarily 
A
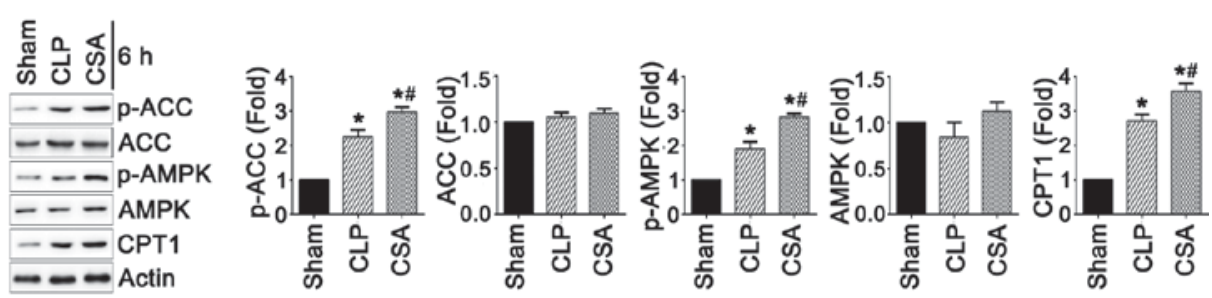

B
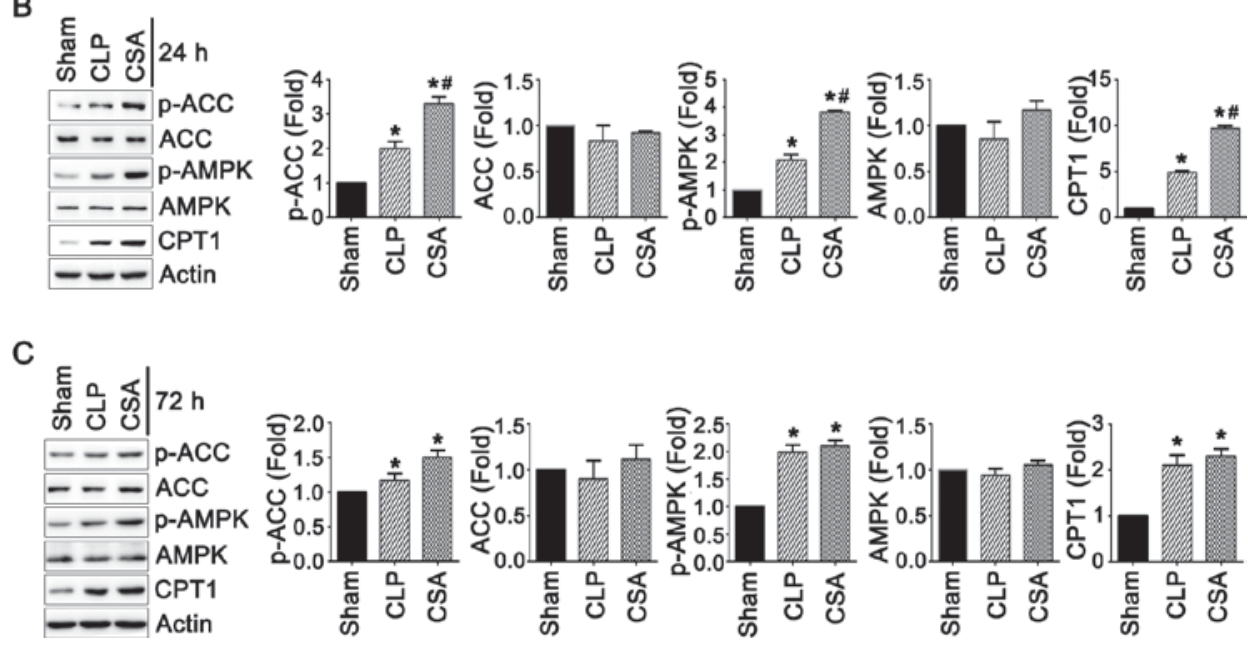

Figure 4. CSA treatment activated the AMPK-ACC-CPT1 signaling pathway. Western blots were performed using heart tissue protein lysates from rats in the sham, CLP and CSA groups to detect levels of the AMPK-ACC-CPT1 pathway components at (A) $6 \mathrm{~h}$, (B) $24 \mathrm{~h}$ and (C) $72 \mathrm{~h}$. Actin was used as a loading control. p-AMPK and p-ACC levels but not total AMPK and ACC levels were significantly elevated by CSA treatment. Data are presented as the mean \pm standard deviation $(n=6)$. ${ }^{*} \mathrm{P}<0.05$ vs. sham; ${ }^{*} \mathrm{P}<0.05$ vs. CLP. p-AMPK, phosphorylated AMP-activated protein kinase; $\mathrm{p}-\mathrm{ACC}$, phosphorylated acetyl CoA carboxylase; CPT1, carnitine palmitoyl transferase 1; sham, sham-operated rats; CLP, cecal ligation puncture procedure sepsis model group; CSA, cyclosporine A-treated sepsis model group.

obtained via mitochondrial oxidative metabolism, where fatty acid oxidation contributes $60-80 \%$ of the energy generated, whereas pyruvate oxidation generates $10-40 \%$ and glycolysis contributes the remainder $(30,31)$. However, during the development of sepsis, glycolysis, instead of free fatty acid oxidation (using NEFA), serves as the major source of energy, which leads to the accumulation of NEFA in cardiomyocytes. Excessive NEFA accumulation in the heart induces the inhibition of mitochondrial oxidative phosphorylation and adenosine triphosphate (ATP)-to-inorganic phosphate conversion, leading to heart damage $(32,33)$. However, how NEFA metabolism is regulated in the myocardium of rats in the septic model is unknown. In the present study, the CLP group exhibited elevated concentrations of NEFA in the plasma and an increased accumulation of NEFA in cardiomyocytes at 6-24 h following sepsis induction when compared with the sham group. Notably, CSA treatment decreased NEFA accumulation in cardiomyocytes at 6 , 12 and $24 \mathrm{~h}$. However, no significant effects on plasma NEFA concentrations were observed at any of the time points examined. In cardiomyocytes, a decrease in NEFA accumulation was observed alongside the recovery of cardiac function achieved by CSA treatment. These findings indicated that the CSA-induced improvement in cardiac function in the rat sepsis model may be partially reduced following NEFA storage in cardiomyocytes. In addition, the increase in the calcineurin activity in plasma observed in rats with sepsis may have been induced via different mechanisms compared with calcineurin induction in the heart and were therefore CSA-independent. As CSA efficiently decreased NEFA accumulation in cardiomyocytes but not in plasma, and CSA is a specific inhibitor for calcineurin, it is possible that increased NEFA accumulation in cardiomyocytes may involve calcineurin-associated signaling pathways. However, further investigation is required to evaluate this hypothesis.

In the present study, the AMPK-ACC-CPT1 calcineurin-mediated signaling pathway, which is a key pathway for the mediation of energy metabolism (NEFA oxidation in particular) (34), was investigated. Activation of the AMPK pathway in the heart may facilitate NEFA oxidation, thereby improving the energy supply and enhancing cardiac function (35). By contrast, inhibition of the AMPK pathway has been observed to reduce NEFA oxidation, thereby increasing NEFA accumulation and the associated toxicity to cardiomyocytes (36). In addition, NEFA accumulation may induce cardiac endoplasmic reticulum stress, together with decreased ATP production, thereby inducing heart failure (21,37-39). Calcineurin is one of the factors that regulate the AMPKACC-CPT1 pathway (40). Cardiac injuries induced by external stimuli, such as $\mathrm{H}_{2} \mathrm{O}_{2}$-activated calcineurin and repression of the AMPK-ACC-CPT1 pathway, lead to altered lipid metabolism and the accumulation of NEFA $(3,40)$. By contrast, inhibition of calcineurin through the use of inhibitors, such as CSA and FK506, has been observed to improve the activity of AMPK-ACC-CPT1 signaling and alleviate myocardial 
injury $(3,40)$. The present study demonstrated that, during the early stages of sepsis, the total protein levels of AMPK and ACC were not significantly altered. However, the levels of their activated forms, $\mathrm{p}$-AMPK and p-ACC, were significantly increased in the initial $72 \mathrm{~h}$-period following sepsis induction. In addition, a similar increase in the protein levels of CPT1, was observed. However, the sepsis-induced activation of the AMPK-ACC-CPT1 pathway was not sufficient to inhibit NEFA accumulation. By contrast, CSA treatment increased the activity of this pathway, which was associated with a significant reduction in NEFA accumulation. Therefore, sepsis may alter lipid metabolism in the heart, thereby activating calcineurin and the AMPK-ACC-CPT1 pathway simultaneously. However, in the presence of activated calcineurin activity, AMPK-ACC-CPT1 signaling may have been unable to facilitate the efficient mobilization of NEFA metabolism in the myocardium. CSA treatment may have partially diminished NEFA accumulation by further upregulating the activity of the AMPK-ACC-CPT1 pathway. Of particular note, the myocardial function in rats with sepsis was comparable to rats in the sham group at $72 \mathrm{~h}$, whereas calcineurin activity and NEFA concentration in the myocardium of rats in the CLP group remained significantly higher at the same time point, which suggests that there may be alternative mechanisms involved in mediating myocardial function at the early stages of sepsis progression.

In conclusion, the results of the present study indicate that sepsis may induce cardiac dysfunction, calcineurin activation and abnormal lipid metabolism. Improved cardiac function by CSA was evidenced by the observed decrease in calcineurin activity and reduced NEFA accumulation in cardiomyocytes. Calcineurin and AMPK signaling are involved in altered lipid metabolism, and CSA reduced NEFA accumulation in the myocardium potentially via partial suppression of calcineurin activity and increased AMPK signaling. Therefore, the results of the present study provide a greater insight into the mechanisms by which CSA is able to alleviate myocardial repression induced by sepsis. In addition, the results present a potential therapeutic treatment for sepsis-induced cardiac dysfunction, at least at the early stages of infection.

\section{References}

1. Iskander KN, Osuchowski MF, Stearns-Kurosawa DJ, Kurosawa S, Stepien D, Valentine C and Remick DG: Sepsis: Multiple abnormalities, heterogeneous responses, and evolving understanding. Physiol Rev 93: 1247-1288, 2013.

2. Singer M, Deutschman CS, Seymour CW, Shankar-Hari M, Annane D, Bauer M, Bellomo R, Bernard GR, Chiche JD, Coopersmith CM, et al: The third international consensus definitions for sepsis and septic shock (Sepsis-3). JAMA 315: 801-810, 2016.

3. Park HG, Yi H, Kim SH, Yu HS, Ahn YM, Lee YH, Roh MS and Kim YS: The effect of cyclosporine A on the phosphorylation of the AMPK pathway in the rat hippocampus. Prog Neuropsychopharmacol Biol Psychiatry 35: 1933-1937, 2011.

4. Parrillo JE: The cardiovascular pathophysiology of sepsis. Annu Rev Med 40: 469-485, 1989.

5. dos Santos CC, Gattas DJ, Tsoporis JN, Smeding L, Kabir G, Masoom H, Akram A, Plotz F, Slutsky AS, Husain M, et al: Sepsis-induced myocardial depression is associated with transcriptional changes in energy metabolism and contractile related genes: A physiological and gene expression-based approach. Crit Care Med 38: 894-902, 2010.

6. Remick DG: Pathophysiology of sepsis. Am J Pathol 170: 1435-1444, 2007.
7. Bueno OF, van Rooij E, Molkentin JD, Doevendans PA and De Windt LJ: Calcineurin and hypertrophic heart disease: Novel insights and remaining questions. Cardiovasc Res 53: 806-821, 2002.

8. Molkentin JD, Lu JR, Antos CL, Markham B, Richardson J, Robbins J, Grant SR and Olson EN: A calcineurin-dependent transcriptional pathway for cardiac hypertrophy. Cell 93: 215-228, 1998.

9. Berry JM, Le V, Rotter D, Battiprolu PK, Grinsfelder B, Tannous P, Burchfield JS, Czubryt M, Backs J, Olson EN, et al: Reversibility of adverse, calcineurin-dependent cardiac remodeling. Circ Res 109: 407-417, 2011.

10. Sussman MA, Lim HW, Gude N, Taigen T, Olson EN, Robbins J, Colbert MC, Gualberto A, Wieczorek DF and Molkentin JD: Prevention of cardiac hypertrophy in mice by calcineurin inhibition. Science 281: 1690-1693, 1998.

11. Suzuki J, Bayna E, Li HL, Molle ED and Lew WY: Lipopolysaccharide activates calcineurin in ventricular myocytes. J Am Coll Cardiol 49: 491-499, 2007.

12. Larche J, Lancel S, Hassoun SM, Favory R, Decoster B, Marchetti P, Chopin C and Neviere R: Inhibition of mitochondrial permeability transition prevents sepsis-induced myocardial dysfunction and mortality. J Am Coll Cardiol 48: 377-385, 2006.

13. Rudiger A and Singer M: Mechanisms of sepsis-induced cardiac dysfunction. Crit Care Med 35: 1599-1608, 2007.

14. Iwamura $\mathrm{H}$, Sato $\mathrm{M}$ and Wakitani $\mathrm{K}$ : Comparative study of glucocorticoids, cyclosporine A, and JTE-607 [(-)-Ethyl-N [3,5-dichloro-2-hydroxy-4-[2-(4-methylpiperazin-1-yl)ethoxy] benzoyl]-L-phenylalaninate dihydrochloride] in a mouse septic shock model. J Pharmacol Exp Ther 311: 1256-1263, 2004.

15. Joshi MS, Julian MW, Huff JE, Bauer JA, Xia Y and Crouser ED: Calcineurin regulates myocardial function during acute endotoxemia. Am J Respir Crit Care Med 173: 999-1007, 2006.

16. Fauvel H, Marchetti P, Obert G, Joulain O, Chopin C, Formstecher $\mathrm{P}$ and Nevière R: Protective effects of cyclosporin A from endotoxin-induced myocardial dysfunction and apoptosis in rats. Am J Respir Crit Care Med 165: 449-455, 2002.

17. $\mathrm{Zu} \mathrm{L}, \mathrm{He}$ J, Jiang H, Xu C, Pu S and Xu G: Bacterial endotoxin stimulates adipose lipolysis via toll-like receptor 4 and extracellular signal-regulated kinase pathway. J Biol Chem 284: 5915-5926, 2009.

18. Heidrich F, Schotola H, Popov AF, Sohns C, Schuenemann J, Friedrich M, Coskun KO, von Lewinski D, Hinz J, Bauer M, et al: AMPK-activated protein kinase and its role in energy metabolism of the heart. Curr Cardiol Rev 6: 337-342, 2010.

19. Fillmore $\mathrm{N}$ and Lopaschuk GD: Targeting mitochondrial oxidative metabolism as an approach to treat heart failure. Biochim Biophys Acta 1833: 857-865, 2013.

20. Kiuchi S, Matsuo N, Takeyama N and Tanaka T: Accelerated hepatic lipid synthesis in fasted septic rats. Eur Surg Res 25: 146-154, 1993.

21. Borradaile NM, Han X, Harp JD, Gale SE, Ory DS and Schaffer JE: Disruption of endoplasmic reticulum structure and integrity in lipotoxic cell death. J Lipid Res 47: 2726-2737, 2006.

22. Son NH, Park TS, Yamashita H, Yokoyama M, Huggins LA, Okajima K, Homma S, Szabolcs MJ, Huang LS and Goldberg IJ: Cardiomyocyte expression of PPARgamma leads to cardiac dysfunction in mice. J Clin Invest 117: 2791-2801, 2007.

23. Torac E, Gaman L and Atanasiu V: The regulator of calcineurin (RCAN1. an important factor involved in atherosclerosis and cardiovascular diseases development. J Med Life 7: 481-487, 2014.

24. Kuwabara Y, Horie T, Baba O, Watanabe S, Nishiga M, Usami S, Izuhara M, Nakao T, Nishino T, Otsu K, et al: MicroRNA-451 exacerbates lipotoxicity in cardiac myocytes and high-fat diet-induced cardiac hypertrophy in mice through suppression of the LKB1/AMPK pathway. Circ Res 116: 279-288, 2015.

25. Ahrens T: Hemodynamics in sepsis. AACN Adv Crit Care 17: 435-445, 2006.

26. Merx MW and Weber C: Sepsis and the heart. Circulation 116: 793-802, 2007

27. Zaky A, Deem S, Bendjelid $K$ and Treggiari MM: Characterization of cardiac dysfunction in sepsis: An ongoing challenge. Shock 41: 12-24, 2014.

28. Molnar AO, Fergusson D, Tsampalieros AK, Bennett A, Fergusson N, Ramsay T and Knoll GA: Generic immunosuppression in solid organ transplantation: Systematic review and meta-analysis. BMJ 350: h3163, 2015.

29. Kakihana Y, Ito T, Nakahara M, Yamaguchi K and Yasuda T: Sepsis-induced myocardial dysfunction: Pathophysiology and management. J Intensive Care 4: 22, 2016. 
30. Stanley WC, Lopaschuk GD, Hall JL and McCormack JG: Regulation of myocardial carbohydrate metabolism under normal and ischaemic conditions. Potential for pharmacological interventions. Cardiovasc Res 33: 243-257, 1997.

31. van der Vusse GJ, Glatz JF, Stam HC and Reneman RS: Fatty acid homeostasis in the normoxic and ischemic heart. Physiol Rev 72: 881-940, 1992.

32. Ebong IA, Goff DC Jr, Rodriguez CJ, Chen H and Bertoni AG: Mechanisms of heart failure in obesity. Obes Res Clin Pract 8: e540-e548, 2014.

33. Haffar T, Bérubé-Simard FA and Bousette N: Cardiomyocyte lipotoxicity is mediated by Il- 6 and causes down-regulation of PPARs. Biochem Biophys Res Commun 459: 54-59, 2015.

34. Srivastava RA, Pinkosky SL, Filippov S, Hanselman JC, Cramer CT and Newton RS: AMP-activated protein kinase: An emerging drug target to regulate imbalances in lipid and carbohydrate metabolism to treat cardio-metabolic diseases. J Lipid Res 53: 2490-2514, 2012.

35. Viollet B and Andreelli F: AMP-activated protein kinase and metabolic control. Handb Exp Pharmacol 303-330, 2011.
36. Park TS, Hu Y, Noh HL, Drosatos K, Okajima K, Buchanan J, Tuinei J, Homma S, Jiang XC, Abel ED and Goldberg IJ: Ceramide is a cardiotoxin in lipotoxic cardiomyopathy. J Lipid Res 49: 2101-2112, 2008.

37. Borradaile NM, Buhman KK, Listenberger LL, Magee CJ, Morimoto ET, Ory DS and Schaffer JE: A critical role for eukaryotic elongation factor 1A-1 in lipotoxic cell death. Mol Biol Cell 17: 770-778, 2006.

38. Song XJ, Yang CY, Liu B, Wei Q, Korkor MT, Liu JY and Yang P: Atorvastatin inhibits myocardial cell apoptosis in a rat model with post-myocardial infarction heart failure by downregulating ER stress response. Int J Med Sci 8: 564-572, 2011.

39. Turner MD: Fatty acyl CoA-mediated inhibition of endoplasmic reticulum assembly. Biochim Biophys Acta 1693: 1-4, 2004.

40. He H, Liu X, Lv L, Liang H, Leng B, Zhao D, Zhang Y, Du Z, Chen X, Li S, et al: Calcineurin suppresses AMPK-dependent cytoprotective autophagy in cardiomyocytes under oxidative stress. Cell Death Dis 5: e997, 2014. 